\title{
ASPECTOS LEGALES Y BIOÉTICOS DE INTERVENCIONES E INVESTIGACIONES EN PERSONAS CON DISCAPACIDAD INTELECTUAL EN CHILE
}

\begin{abstract}
María Teresa Muñoz Quezada ${ }^{1}$, Boris Andrés Lucero Mondaca ${ }^{1}$
Resumen: El trabajo presenta una revisión y discusión de los aspectos legales y éticos que protegen a los sujetos con discapacidad intelectual en la participación de investigaciones o intervenciones terapéuticas en Chile, además de reflexionar sobre situaciones de diagnóstico y tratamiento que generan conflicto respecto de los derechos de las personas con discapacidad intelectual. Los temas específicos se vinculan con la decisión autónoma y capacidad en el consentimiento informado, los derechos de las personas con discapacidad intelectual, la evaluación de la capacidad y la reflexión sobre algunos dilemas bioéticos en el tratamiento y evaluación de la discapacidad intelectual. Se propone reconocer el derecho de las personas con discapacidad intelectual a decidir por sí mismos y ser reconocidos en igualdad de oportunidades y derechos, desarrollar procedimientos y protocolos para la evaluación de la capacidad estandarizados y adaptados a la diversidad cultural del país, y que cuidadores, familiares y profesionales consideren no solo las normativas frente a la investigación y tratamiento, sino también la opinión y decisión de las personas con discapacidad, e informarles en qué consisten dichas experiencias, considerando sus características individuales.
\end{abstract}

Palabras clave: bioética, discapacidad intelectual, consentimiento informado

\section{Legal and bioethical issues of interventions and research in persons with intellectual disability in Chile}

\begin{abstract}
This article presents a review and discussion of ethical and legal issues which protect subjects with intellectual disability in their participation in research or therapeutic interventions in Chile, moreover reflecting on situations of diagnosis and treatment which generate conflict with respect to the rights of persons with intellectual disability.

The specific topics are linked to autonomous decision and capacity in informed consent, the rights of persons with intellectual disability, the evaluation of capacity and the reflection on some bioethical dilemmas in the treatment and evaluation of intellectual disability. The following is proposed: to recognize the rights of persons with intellectual disability to decide by themselves and the recognition of equal rights and opportunities to develop procedures and protocols standardized and adapted for cultural diversity in the country for evaluating capacity. Furthermore, caretakers, family members and professionals should consider not only regulations for research and treatment, but also the opinion and decision of persons with disabilities and inform them about such experiences considering their individual characteristics.
\end{abstract}

Key words: Bioethics, intellectual disability, informed consent

\section{Aspectos legais e bioéticos de intervençóes e pesquisas em pessoas com descapacidade intelectual no Chile}

Resumo: O trabalho apresenta uma revisão e discussão dos aspectos legais e éticos que protegem os sujeitos com descapacidade intelectual na participação de investigações ou intervenções terapêuticas no Chile, além de refletir sobre situaçôes de diagnóstico e tratamento que geram conflito a respeito dos direitos das pessoas com descapacidade intelectual.

Os temas específicos se vinculam com a decisão autônoma e capacidade do consentimento informado, os direitos das pessoas com descapacidade intelectual, a avaliação da capacidade e a reflexão sobre alguns dilemas bioéticos no tratamento e avaliaçáo da descapacidade intelectual. Propóe-se reconhecer o direito das pessoas com descapacidade intelectual para decidir por si mesmas e ser reconhecidas em igualdade de oportunidades e direitos, desenvolver procedimentos e protocolos padróes para a avaliação da capacidade e adaptados à diversidade cultural do país, e que cuidadores, familiares e profissionais considerem não só as normativas frente à investigaçáo e tratamento, mas também a opinião e decisão das pessoas com descapacidade, e informar-lhes em que consistem as ditas experiências, considerando suas características individuais.

Palavras-chave: bioética, descapacidade intelectual, consentimento informado

\footnotetext{
${ }^{1}$ Departamento de Psicología, Universidad Católica del Maule, Chile Correspondencia: mtmunoz@ucm.cl
} 


\section{Introducción}

Los códigos de ética y las leyes regulan el estudio o intervención en aquellos grupos que se consideran vulnerables - niños, adultos no competentes, individuos con trastornos mentales (incluye a las personas con discapacidad intelectual), personas privadas de libertad e internadas en instituciones cerradas. Sin embargo, existen ciertas condiciones para la realización de intervenciones en grupos vulnerables: cuando el objetivo sea obtener conocimiento pertinente a sus necesidades de salud y que no pueda obtenerse por otros medios, y cuando su representante legal, cuando corresponda, haya dado consentimiento libre e infor$\operatorname{mado}(1-6)$.

Teniendo presente lo anterior y considerando las convenciones de derechos humanos de las personas con discapacidad, existe explícita prohibición de realizar investigaciones biomédicas en personas física y psíquicamente incapaces. Si un profesional no respeta el proceso de consentimiento informado, arriesga penas legales e incluso el término de su ejercicio profesional $(1,2)$.

Por lo tanto, el hecho de decidir sobre la participación en una investigación o de una intervención clínica estará vinculada a la capacidad y voluntariedad informada de una persona.

Sin embargo, este proceso suele ser más complejo, sobre todo si se tienen presente los derechos de las personas con discapacidad, que exigen se respete su posibilidad de tomar decisiones en situaciones clínicas, sociales y económicas que les acontecen(7-9). Además, se suma la dificultad de llegar a un consenso en los procesos de evaluación de capacidad que no vulnere la autonomía de las personas.

El objetivo del siguiente trabajo consiste en revisar y discutir los aspectos legales y bioéticos en los procesos de investigación e intervención de las personas con discapacidad intelectual, además de reflexionar sobre algunas situaciones conflictivas respecto de sus derechos.

\section{Método}

Se realizó una revisión de literatura científica en las bases de datos electrónicas EBSCOhost, PubMed y SciELO, libros de divulgación científica, documentos de convenios, normativas y leyes, páginas web de instituciones públicas y privadas, y artículos de ética vinculados con la temática de consentimiento informado y discapacidad intelectual. Se analizaron los contenidos de los documentos y la información se organizó en tres temáticas relevantes: decisión autónoma y capacidad en el consentimiento informado, evaluación de la capacidad de consentimiento informado y algunos dilemas éticos.

\section{Resultados}

Decisión autónoma y capacidad en el consentimiento informado.

El consentimiento informado es un proceso que involucra al investigador o terapeuta y al participante o paciente, abarcando toda la investigación o intervención y no solo el comienzo de esta. Incluye la entrega de información sobre el estudio o tratamiento de manera comprensible para los sujetos, dar respuesta a sus preguntas, posibilitar el tiempo suficiente para que examinen sus decisiones y obtener un acuerdo voluntario y autóno$\operatorname{mo}(4,10,11)$.

Los elementos del consentimiento informado para las investigaciones comprenden: describir la investigación, mencionar y explicar riesgos y beneficios, especificar alternativas de participación, explicar la confidencialidad, expresar las normas de compensación, el contacto con los responsables de la investigación y la explicitación de la voluntariedad de ingresar y continuar en el estu$\operatorname{dio}(10)$.

Los aspectos del consentimiento en intervenciones se relacionan con la autonomía del paciente, decidir si acepta o no las condiciones de riesgos y beneficios que trae el tratamiento, sujeta a la capacidad de tomar decisiones, de manera libre y voluntaria, y que dicha decisión se adopte tras comprender y evaluar la información recibida de parte del profesional tratante(11).

En Chile el consentimiento informado tiene varias regulaciones legales. El Código Civil chileno(12) entiende como capacidad legal la posibilidad de 
una persona de obligarse por sí misma y sin la autorización de otra. Toda persona es legalmente capaz, excepto aquellas que la ley declara incapaces, y son incapaces absolutos los dementes, los impúberes y los sordomudos que no pueden darse a entender por escrito, todos quienes necesitan un representante legal, cuyas acciones tendrán el mismo efecto que si hubiese consentido la misma persona representada.

La Ley chilena 20.120, sobre la investigación científica en seres humanos, menciona que el estudio científico debe contar con consentimiento previo, libre e informado; el sujeto que lo prestará conocerá la finalidad, beneficios, riesgos y los procedimientos o tratamientos alternativos de la investigación. Si un investigador no respetare el consentimiento, arriesga sanciones penales, y si reincide puede ser sancionado con la prohibición total de ejercicio profesional(1).

La posibilidad de participar en una investigación o de entregar el consentimiento para una intervención dependerá de la autonomía y capacidad de las personas en la toma de decisiones.

Beauchamp y Childress(13) proponen la autonomía como unos de los principios bioéticos de la práctica clínica y en la investigación con seres humanos, entendiéndola como respeto por las decisiones de otros y por las opiniones y actuar propios, tanto en relación con valores como con creencias personales. Para que este principio sea útil debe estar vinculado a un contexto que entregue derechos y obligaciones de libertad y confidencialidad para el consentimiento. En el caso de adultos con discapacidad intelectual, la autonomía estaría relacionada con la capacidad de decidir frente a las situaciones que impliquen o no un riesgo hacia la persona. Los autores argumentan que el principio de autonomía puede ser superado por otras condiciones morales en el caso de que la persona tome una decisión que implique un daño para sí mismo, lo que se vincula con el principio de beneficencia, que involucra la obligación de promover los derechos de otro, prevenir situaciones de peligro y, en este contexto, ayudar a las personas con discapacidad.

El Código de Ética del Colegio Médico chileno especifica que el consentimiento expreso deberá ser entregado por el enfermo o familiares responsables - en el caso de menores de edad o incapacidad - cuando las intervenciones signifiquen riesgos ciertos o mutilación grave para el paciente, y, si no hay representante, el médico entregará los cuidados que le dicte su conciencia $(2,14)$.

El Código de Ética del Colegio de Psicólogos chileno(15) estipula que en las investigaciones el profesional debe seguir las normas y criterios de la comunidad científica, además de resguardar el bienestar y derechos de los participantes. Declara que el consentimiento escrito, tanto para investigaciones como para tratamientos, deberá ser entregado por personas con discernimiento, o por su representante legal, informando de los posibles riesgos o efectos adversos a los participantes o sus representantes.

En los códigos de ética consultados se observa que la definición de "capacidad" se asimila con la de "competencia". Este aspecto ya había sido tratado por otros autores que discutían sobre la complejidad del término "capacidad" y el uso que se le ha dado como sinónimo de "competencia" en los contextos clínicos(2,16-19). Esto se suma a la ausencia, en los códigos de ética y normativas de investigación, de orientaciones explícitas sobre la evaluación de la capacidad para entregar el consentimiento.

La complejidad para entender los términos de "capacidad" y "competencia" emerge desde su traducción. "Capacidad" se define como la aptitud o cualidad que dispone a un individuo para el buen ejercicio de algo. Se distingue la capacidad de obrar - que sería la aptitud para ejercer un derecho y cumplimiento de una obligación- de la capacidad jurídica — referida a la aptitud legal para ser sujeto de derechos y obligaciones. Por "competencia" se entiende la aptitud o idoneidad para actuar o intervenir en alguna situación, o la atribución legítima de un juez para la resolución de un asunto $(16,19)$. En la literatura anglosajona la capacidad jurídica o de derecho se refiere al término "competencia", en cambio la capacidad propiamente tal se relaciona con la capacidad de hecho o natural. En Chile, en el ámbito clínico o de investigación, se utilizan como sinónimos los conceptos de "competencia" y "capacidad" $(16,17)$. 
Según Beauchamp y Childress(13) la competencia se refiere a la capacidad para realizar una tarea, y la función que cumple es diferenciar entre quienes pueden decidir frente a una situación (competentes) y quienes no pueden obtener respeto de sus decisiones (incompetentes). Esto es relativo al contexto; por ejemplo, una persona puede ser competente para decidir frente a un tratamiento o investigación, pero no en el manejo de sus finanzas. Por lo tanto, los autores proponen estándares de competencia según la tarea propuesta. De aquí emergen una serie de instrumentos para evaluar la capacidad o "competencia" de un individuo para consentir, los que serán descritos más adelante.

Proceder a determinar la competencia de una persona en la toma decisiones depende de una serie de etapas $(13,16,19,20)$. Poseer capacidad de hecho o natural determina la capacidad de derecho. Si se observa que un sujeto presenta dificultades en esta condición puede dar paso a evaluar una incapacidad de hecho transitoria o una incapacidad de hecho permanente. Una incapacidad transitoria derivaría en una incapacidad de derecho transitoria que, si persiste, el sujeto puede ser declarado como incapaz o incompetente momentáneamente. En cambio, cuando se presenta una incapacidad de hecho permanente se realiza el procedimiento de incapacitación o interdicción por presunta demencia, se solicitan peritajes a médicos especialistas para determinar el diagnóstico y, si se confirma, deriva a una incapacidad de derecho permanente y a la condición de incapacitado absoluto(13). Este estado legal obligaría a un adulto con discapacidad intelectual a no poder participar en la toma de decisiones.

El hecho de declarar demente a un adulto con discapacidad intelectual confunde a los personas que presentan dicho diagnóstico y a sus familiares. La discapacidad intelectual se define como un trastorno de inicio del desarrollo, que incluye déficits de funcionamiento intelectual y adaptativo en los dominios conceptual, social y práctico $(21,22$ 24,25), y se entiende como producto de una o más limitaciones psíquicas, congénitas o adquiridas, relativamente permanente, independiente de la causa que la originó, limitando en al menos un tercio la capacidad educativa, laboral o de integración social, con un rendimiento igual o inferior al $70 \%$ de lo esperado para una persona de igual edad y condición social y cultural, medido por un instrumento validado por la Organización Mundial de la Salud (OMS) y administrado individualmente $(21,24,25)$.

Con la inserción del modelo multidimensional de discapacidad intelectual de la Asociación Americana de Discapacidad Intelectual y del Desarrollo (AAIDD), de la OMS y la Asociación Americana de Psiquiatría (APA), el concepto y diagnóstico de "retraso mental" fue reformulado por el de "discapacidad intelectual"(25-28), incorporando las potencialidades de las personas más allá de su coeficiente intelectual, definiéndolo como una condición variable que va modificándose en el desarrollo del individuo, por la disponibilidad de apoyos que recibe y por la interacción con el entorno sociocultural.

Actualmente, el proceso de evaluación de la discapacidad intelectual lo realizan psicólogos y médicos especializados. Este procedimiento involucra el diagnóstico, clasificación y descripción, es decir, determina los aspectos fuertes y débiles del sujeto en diversas dimensiones, y propone un perfil de necesidades de apoyo $(21,25-29)$.

Para diagnosticar discapacidad intelectual deben presentarse tres criterios(25): limitaciones significativas en el funcionamiento intelectual y en la conducta adaptativa, y el inicio de las deficiencias intelectuales y de adaptación durante el periodo de desarrollo.

Este modelo pretende evaluar y describir el desenvolvimiento en diversas áreas: habilidades intelectuales, conducta adaptativa, participación, salud y contexto ambiental y cultural (24-28). La evaluación y clasificación en estas cinco dimensiones tienen como finalidad concretar los apoyos que requiere el individuo con discapacidad intelectual, para favorecer su funcionamiento en un contexto sociocultural. Por otro lado, este modelo multidimensional adopta una postura constructiva, considerando una evaluación integral, que orienta a establecer soluciones concretas en cada dimensión y a revisar permanentemente las intervenciones que se generan para modificar o mejorar dicha condición. Al mismo tiempo, limita el abuso del diagnóstico determinado por el coeficiente intelectual, aspecto que ha sido am- 
pliamente debatido en los casos de discapacidad intelectual leve, cuando el diagnóstico emerge de espacios socioculturales diferentes de los que provienen los instrumentos estandarizados en la cultura dominante(25-30).

Se suman a esta reformulación los esfuerzos realizados por agrupaciones que defienden los derechos de personas con discapacidad intelectual y las políticas y normativas internacionales, que apuntan a una visión normalizadora, orientada a la autonomía y a la protección basada en derechos $(31,32)$. En estos esfuerzos destacan la convención de Derechos de Personas con Discapacidad(8) y la Declaración de Montreal sobre la Discapacidad Intelectual(9).

La Declaración de Montreal parte de la visión de que las personas con discapacidad intelectual, como todo ser humano, nacen libres e iguales en dignidad y derechos que, al igual que otras características humanas, son parte integral de la experiencia y diversidad. Se explicita que los Estados deben garantizar la protección de las personas con esa condición en la experimentación médica o científica, informando y velando porque reciban y tengan acceso a los servicios que requieran según sus necesidades, y a participar en el proceso de consentimiento. Por lo tanto, expresa que estas personas tienen derecho a tomar decisiones sobre sus vidas.

Esto es un cuestionamiento a las visiones que los determinan con deficientes habilidades cognitivas para comprender los tratamientos a los que serán sometidos, y orienta a una revisión de las posibilidades reales de voluntariedad y autonomía en sus experiencias cotidianas. Probablemente, esta perspectiva tiene un mayor argumento en los casos de discapacidades intelectuales leves o moderadas, pero no queda claro qué ocurre con las discapacidades graves y profundas.

Según esta declaración, quienes presentan una discapacidad intelectual y dificultades para realizar elecciones, nunca serán consideradas totalmente incompetentes para tomar decisiones. En casos excepcionales, este derecho puede ser interrumpido, pero debe ser limitado en el tiempo y sujeto a revisiones periódicas y no generalizadas a otros ámbitos de su vida; es decir, un adulto con discapacidad intelectual grave tal vez no pueda manejar sus bienes, pero eso no significa que no se puedan agotar diversos medios para que comprenda la situación legal que vivencia o que pueda decidir o expresar su opinión sobre la aplicación de algún tratamiento o investigación en su persona.

Se propone, además, que sea una autoridad competente e independiente quien presente evidencias claras y convincentes de que la persona posee una capacidad limitada para la toma de decisiones, quien debe actuar según la ley y respetando el derecho de la persona a ser informada de los procedimientos, a emitir su opinión y a ser representada por los individuos que ella decida y en quienes confíe. El representante a cargo del consentimiento sustitutivo deberá tomar en cuenta las preferencias de la persona y hacer todo lo posible por decidir lo que esta escogería si pudiera hacerlo por sí misma.

Este punto tiene una gran complejidad en los adultos con discapacidad intelectual grave y profunda, sobre todo en los casos en que existen graves trastornos de la comunicación, en los que tomar una decisión suponiendo lo que esa persona hubiera hecho es bastante cuestionable y quedaría al criterio de quien decide. En esas situaciones debiera existir, además, una revisión exhaustiva de un comité de expertos externos que evalúen la elección final.

\section{Evaluación de la capacidad de consentimiento in- formado}

En algunas naciones se aplican escalas que evalúan la capacidad de un paciente para dar el consentimiento informado. En Chile, la evaluación de la capacidad es subjetiva, de responsabilidad de algunos profesionales de la salud, recurriendo en ocasiones a expertos quienes definen un diagnóstico de salud mental y no un juicio de capacidad para participar en un estudio o en el desarrollo de una intervención $(16,17)$.

La complejidad de la evaluación de la capacidad para dar el consentimiento informado ha sido abordada en diversas investigaciones sobre demencia y discapacidad intelectual(19,33-36). En Chile, Bórquez et al.(17) han identificado que los 
médicos evalúan la capacidad con una entrevista clínica, reconociendo inexistencia de instrumentos y procedimientos estandarizados. Algunos de los indicadores utilizados son las habilidades de razonamiento, conciencia, discernimiento, capacidad intelectual, afectiva y emocional, capacidad de autonomía y autocontrol. Solamente especialistas de adolescentes y adultos mayores aplican escalas de evaluación de la capacidad. Los psiquiatras utilizan el examen clínico, no encontrándose coincidencia en el uso de instrumentos. Se menciona que la necesidad de evaluación es frecuente, pero se realiza de manera informal y variada según la especialidad. En este sentido, los autores expresan que esta diversidad puede generar discriminación y daño para la persona supuestamente incapaz como, al mismo tiempo, falta de protección de una persona que se sugiere capaz y no lo es.

Para la evaluación de la capacidad existen diversos instrumentos, como las pruebas neuropsicológicas, cuestionarios generales de evaluación de la capacidad y protocolos específicos de capacidad(18). Algunos ejemplos de protocolos:

1. California Scale of Appreciation (CSA) (37), permite evaluar capacidad para participar en investigaciones. Consiste en una entrevista semiestructurada y se puntúa $O=\operatorname{Sin}$ capacidad 1 = Capacidad relativa; 2 = Capacidad . El instrumento consta de 13 preguntas cerradas en las cuales la persona debe contestar verdadero o falso, y posee 5 preguntas abiertas. Algunos ejemplos de preguntas cerradas: "Nada terrible me pasará si decido estar o no en este estudio" (pregunta 1), "El investigador tiene habilidades especiales o poderes que me protegerán de todo daño" (pregunta 4). Ejemplos de las preguntas abiertas serían "¿Cree que este estudio es solamente una investigación o solamente un tratamiento? ¿Por qué?” (pregunta 1), “Por qué cree usted que se le pregunta su participación en este estudio?” (pregunta 4).

2. MacArthur Competence Assessment Tool (McCAT) (19), contiene una entrevista semiestructurada. El McCAT para participar en proyectos de investigación contiene además la evaluación de una situación. El McCAT que evalúa capacidad para dar consentimiento de una intervención considera los criterios de comprensión de la enfermedad, del tratamiento y de los riesgos y beneficios; apreciación de la situación de la enfermedad y del objetivo del tratamiento; razonamiento, y si es capaz de expresar una decisión.

3. Documento Sitges (36), consiste en un protocolo que evalúa la capacidad para dar consentimiento informado en seis situaciones distintas: decisiones que influyen en la propia salud, sobre la participación en investigaciones, patrimonio, actividades propias no laborales, decisiones sobre sí mismo diferentes de las anteriores y decisiones acerca de otras personas sobre las que se tiene responsabilidad no laboral. Estas situaciones se evalúan con 16 criterios neurológicos, por ejemplo, nivel de conciencia, cálculo, orientación espacial, razonamiento abstracto, entre otros. Cada criterio se evalúa como: 1 Normal; 2 Deterioro ligero; 3 Deterioro moderado; 4 Deterioro intenso; 5 Función abolida.

Por tanto, emerge la complejidad bioética en la evaluación de la capacidad para los procesos de toma de decisiones y consentimiento informado en el contexto clínico y de investigación. En el ámbito científico, se cuestiona la evaluación de la capacidad con pruebas cognitivas, se observan que no son adecuados predictores de la capacidad; por ende, algunos autores proponen generar procedimientos que cumplan con criterios bioéticos y jurídicos $(16,19,33)$.

\section{Algunos dilemas éticos}

Estas situaciones son críticas cuando involucran a adultos con discapacidad intelectual y cuando otros toman decisiones sobre sus tratamientos o requerimientos terapéuticos. Aspectos como la esterilización, evaluaciones para diagnosticar su capacidad intelectual o imposición de ser sujetos de investigación en un supuesto estudio que mejorará su calidad de vida son algunos ejemplos de las situaciones cuestionables en un nivel bioético y que vulneran sus derechos. La Ley 20.584 controla que no se realicen investigaciones con personas con discapacidad que no pueden dar su consentimiento; sin embargo, en intervenciones clínicas aún prevalece el consentimiento del representante legal(2). 
Por ejemplo, la esterilización en adultos con discapacidad intelectual(39), realizada con el fin de evitar un posible embarazo por abuso sexual en situación de internación, prevenir daños en el feto y evitar dificultades para la familia al no asumir la paternidad/maternidad. Si se trata de una persona que es considerada incapaz, será el representante quien otorgará el consentimiento para realizar el procedimiento de esterilización. Si bien requiere de una junta médica que analice el caso y la esterilización se lleva a cabo en situaciones que se consideran de riesgo, muchas veces son los familiares quienes la solicitan para prevenir posibles embarazos y en ocasiones los adultos con discapacidad desconocen el procedimiento realizado o se enteran posteriormente. ¿Corresponde esterilizar a un adulto con discapacidad intelectual sin su consentimiento? ¿La esterilización protege a las personas con discapacidad o a sus familias? ¿Quién y cómo evalúa la capacidad para asumir la paternidad o maternidad? Es evidente que estos procedimientos afectan al adulto con discapacidad intelectual que desea conformar familia, vivir su sexualidad o decidir sobre su propio cuerpo.

Según diversos estudios(40-43), las personas con discapacidad intelectual leve y moderada poseen capacidad para controlar su sexualidad y algunos logran conformar una familia. Se ha constatado también que la conviccion que señala que la esterilización es necesaria en adultos con discapacidad intelectual, debido a conductas sexuales poco adaptadas o exacerbada, es un mito. Posiblemente estén tan expuestos como todo adolescente o adulto; por lo tanto, la importancia de la educación sexual para prevenir situaciones de riesgo o embarazos no deseados es una propuesta de acción que no trasgrede sus derechos y autonomía de decidir sobre su propio cuerpo.

Otro aspecto sujeto a controversia es la evaluación y diagnóstico de discapacidad intelectual. En nuestro país, la pesquisa de discapacidad intelectual se realiza por lo general en las etapas escolares, cuando el niño demuestra un rendimiento más descendido que sus pares. Cuando es diagnosticado con esta condición, es derivado a un proyecto de integración escolar o a una escuela especial. Además, la familia, si es de escasos recursos, puede tramitar la pensión asistencial en la Comisión de Medicina Preventiva y de Invali- $\operatorname{dez}(22)$. Esto deriva que a muy temprana edad se tiende a asumir como permanente el diagnóstico y el niño se ve sometido a una etiqueta difícil de remover, afectando su autoestima y la posibilidad de mejorar sus aprendizajes. Por lo general, el diagnóstico se basa en la dificultad de aprendizaje del estudiante, obviando la diversidad cultural y su adaptación social en el contexto en que habita. Por otro lado, cabe preguntarse si los profesionales que evalúan en escuelas aplican el procedimiento de consentimiento informado como sugieren los códigos de ética, explicando a los padres los riesgos y beneficios sociales, psicológicos y físicos de realizar el diagnóstico y tratamiento, con el fin de que decidan informados y con la orientación pertinente para participar en el tratamiento de su hijo. Esto es difícil de observar y verificar y muchas veces no se mide la consecuencia de un diagnóstico de discapacidad intelectual, transformado en una minusvalía que limita las posibilidades de desarrollo del estudiante(29,30).

\section{Conclusiones}

En síntesis, a partir de la reflexión anterior, es relevante discutir y considerar los siguientes puntos:

- Reconocer el derecho de personas con discapacidad intelectual a decidir por sí mismos, ser reconocidos en igualdad de oportunidades y derechos, es una responsabilidad y compromiso ético de la sociedad, de sus cuidadores y de los profesionales que los atienden, aspecto que contempla la Ley chilena 20.584(2). Pero el control y regulación de su cumplimiento depende de la conducta ética de los profesionales que evalúan la capacidad de autonomía de dichas personas.

- Evaluar la situación de personas con discapacidad intelectual leve o que no poseen grandes dificultades en sus funciones adaptativas pero presentan un rendimiento inferior en pruebas cognitivas. Se sugiere no emitir un diagnóstico o un juicio clínico de incapacidad a partir de los resultados en dichas pruebas, y no tomar decisiones sobre sus acciones o su cuerpo sin consultar y explicar completa y claramente el tratamiento o intervención que se le propone. 
- Es importante generar un espacio que permita discutir la propuesta de sustituir el representante personal (a quien se le transfieren los derechos y decisiones) por un asistente personal (no supone transferencia de derechos y toma de decisiones), quien debería orientar a la persona con discapacidad sobre los tratamientos o intervenciones que va a recibir o la investigación en que va a participar, y si debido a su discapacidad no puede expresar sus opiniones o creencias, solo en ese momento tomar una decisión que no involucre un posicionamiento de su derecho como persona. En este sentido, la sociedad debe favorecer la autonomía, pero al mismo tiempo velar por una protección basada en derechos $(8,9,28,31,32)$. Por otro lado, el riesgo de la desprotección y vulneración de derechos en la investigación de adultos con discapacidad debe ser regulado por entidades neutras avaladas o controladas por el Estado, y hacer efectiva la denuncia de aquellos profesionales que desarrollan estudios con seres humanos sin consentimiento informado. Esto ya es regulado en cierta medida en la Ley 20.584, en la que se explicita la prohibi- ción de realizar investigaciones científicas con aquellas personas que no pueden expresar su voluntad producto de la limitación psíquica o discapacidad intelectual(2).

- Especificar la responsabilidad y rol de la familia y del profesional tratante en el consentimiento informado y en los procedimientos clínicos a los que es sometido el paciente con discapacidad intelectual.

- Desarrollar un procedimiento para la evaluación de la capacidad, estandarizando protocolos y especificándolos en las normativas, reglamentos y decretos, considerando la diversidad cultural y social del país.

- Diferenciar entre el adulto con discapacidad intelectual y la persona que presenta demencia o trastornos psiquiátricos.

- Finalmente, respecto de los profesionales, velar no solo por conocer las normativas y leyes frente a la investigación y tratamiento, sino que también por la realidad e historia de cada persona que se pretende apoyar, guiar o proteger. 


\section{Referencias}

1. Ministerio de Salud. Ley 20.120. Sobre la investigación cientifica en el ser humano, su genoma, y prohibe la clonación humana. Santiago: MINSAL; 2006.

2. Ministerio de Salud. Ley 20.584. Regula los derechos y deberes que tienen las personas en relación con acciones vinculadas a su atención en salud. Santiago: MINSAL; 2012.

3. La Rocca S, Martínez G, Rascio A, Bajardi M. La investigación biomédica y el consentimiento informado en el ámbito de las poblaciones e individuos vulnerables. Acta Bioethica 2005; 11(2): 169-81.

4. Consejo de Organizaciones Internacionales de las Ciencias Médicas (CIOMS). Pautas Éticas Internacionales de las Ciencias Médicas (CIOMS) en colaboración con la Organización Mundial de la Salud-Ginebra 2002. Programa Regional de Bioética OPS/OMS. Santiago: LOM Ediciones; 2003.

5. Arboleda-Florez J. La investigación en sujetos humanos: poblaciones vulnerables. En Lolas F, Quezada A, eds. Pautas éticas de investigación en sujetos humanos. Santiago de Chile: OPS/OMS Serie Publicaciones. Programa Regional de Bioética; 2003: 129-142.

6. Montoya G. Poblaciones especiales en investigación biomédica. En Lolas F, Quezada A, Rodríguez E. Investigación en salud. Dimensión ética. Santiago de Chile: CIEB, Universidad de Chile; 2006: 191-215.

7. Organización de las Naciones Unidas para la Educación, la Ciencia y la Cultura (UNESCO). Declaración Universal sobre Bioética y Derechos Humanos; 2005. [Consultado 22 julio 2009]. Disponible en: http://www.bioeticanet.info/ documentos/DecUBio_DHesp06.pdf

8. Organización de las Naciones Unidas (ONU). Convención sobre los derechos de las personas con discapacidad; 2007. [Consultado 23 julio 2009]. Disponible en http://www.un.org/disabilities/documents/convention/convoptprot-s.pdf

9. Organización Panamericana de la Salud/Organización Mundial de la Salud (OPS/OMS). La declaración de Montreal sobre la discapacidad intelectual; 2004. [Consultado 23 julio 2009]. Disponible en http://www.inclusion-international. org/site_uploads/File/declaracion.sp.pdf

10. Michaud P. Consideraciones sobre el consentimiento informado en investigación bioética. En Kottow M, ed. Bioética e investigación en seres humanos y animales. Santiago: Conicyt; 2006: 33-44.

11. American College of Physicians. Ethics Manual. Ann Intern Med 1998; 128: 576-594.

12. República de Chile. Código Civil de la República de Chile. De los Actos y Declaración de Voluntad. Libro IV Título II. Santiago: República de Chile; 2000. [Consultado 18 julio 2009]. Disponible en http://www.paginaschile.cl/biblioteca_juridica/codigo_civil/libro_cuarto.htm

13. Beauchamp T, Childress J. Principios de ética biomédica. Barcelona: Masson; 1999.

14. Colegio Médico de Chile. Código de Ética. Santiago: Colegio Médico de Chile AG; 2008. [Consultado 12 agosto 2009] Disponible en: http://www.colegiomedico.cl/Portals/0/files/etica/090305codigo_etica.pdf

15. Colegio de Psicólogos de Chile. Código de ética profesional. Santiago: Colegio de Psicólogos de Chile AG; 1998.

16. Bórquez G, Raineri G, Bravo M. La evaluación de la capacidad de la persona: en la práctica actual y en el contexto del consentimiento informado. Rev Med Chile 2004; 132: 1243-48.

17. Bórquez G, Raineri G, Horwitz N, Huepe G. La noción de capacidad de la persona para tomar decisiones, en la práctica médica y legal. Rev Méd Chile 2007; 135: 1153-59.

18. Borquez G, Horwitz N, López I, Raineri G. Capacidad de los pacientes para tomar decisiones en salud. Actitud y significado para médicos y abogados. Acta Bioethica 2008; 14 (2): 166-175.

19. Simón-Lorda P. La capacidad de los pacientes para tomar decisiones: una tarea todavía pendiente. Rev. Asoc. Esp. Neuropsiq. 2008; 28(102): 325-348.

20. Ministerio de Planificación y Cooperación. Ley 19.954. Modifica la Ley 18.600, en lo relativo al procedimiento de interdicción de los discapacitados mentales. Santiago de Chile: MIDEPLAN; 2004.

21. Ministerio de Planificación y Cooperación. Ley 19.735. Modifica la Ley 18.600, estableciendo nuevas normas sobre los discapacitados. Santiago de Chile: MIDEPLAN; 2001.

22. Ministerio de Planificación. Ley 20.422. Establece sobre igualdad de oportunidades e inclusión social de personas con discapacidad. Santiago de Chile: MIDEPLAN; 2011.

23. Organización Mundial de la Salud. CIE-10: Clasificación internacional estadística de enfermedades y problemas de salud. Geneva: Organización Mundial de la Salud; 1999.

24. Organización mundial de la Salud. Clasificación internacional del funcionamiento, de la discapacidad y de la Salud (CIF). Geneva: Organización Mundial de la Salud; 2001.

25. American Psychiatric Association. Diagnostic and statistical manual of mental disorders, Fith Edition. Arlington, VA: American Psychiatric Association; 2013. 
26. Luckasson R, Borthwick-Duffy S, Buntix W, Coulter D, Craig E, Reeve A et al. Mental retardation: Definition, classification, and systems of supports. Washington, DC: American Association on Mental Retardation; 2002.

27. Wehmeyer M, Buntinx W, Lachapelle Y, Luckasson R, Schalock R, Verdugo M. El constructo de discapacidad intelectual y su relación con el funcionamiento humano. Siglo Cero 2008; 39(227): 5-17.

28. Schalock R, Luckasson R, Shogren K, Borthwick-Duffy S, Bradley V, Buntix W et al. The renaming of mental retardation: Understanding the change to the term intellectual disability. AJIDD 2007; 45: 116-124.

29. Klotz J. Sociocultural study of intellectual disability: Moving beyond labelling and social constructionist perspectives. British Journal of Learning Dissabilities 2004; 32: 93-104.

30. Martorell A, Ayuso J. La incertidumbre en la medida de la inteligencia. Actas españolas de psiquiatría 2004; 32(2): 98 106.

31. Peralta M, González-Torres M. El movimiento hacia la autoderterminación personal: antecedentes y estado actual. En Reyes M, Albéniz B, Conejero S. coord. El largo camino hacia una educación inclusiva: la educación especial y social del siglo XIX a nuestros días. XV Coloquio de Historia de la Educación, Pamplona-Iruñea 2009; 1: 181-192.

32. Peralta F, González-Torres M. La autodeterminación en el contexto del retraso mental: ¿De quimera a realidad? Revista Española de Pedagogía 2005; 63(231): 275-288.

33. Warner J, McCarney R, Griffin M, Hill K, Fisher P. Participation in dementia research: rates and correlates of capacity to give informed consent. J Med Ethics 2008; 34: 167-170.

34. Fisher C, Cea C, Davidson P, Fried A. Capacity of Persons with Mental Retardation to Consent to Participation in Randomized Clinical Trials. American Journal of Psychiatry 2006; 163: 1813-1820.

35. Wong J, Clare I, Holland A, Watson P, Gunn M. The Capacity of People with a Mental Disability to Make a Health Care Decision, Psychological Medicine 2000; 30: 295-306.

36. Sturman E. The capacity to consent to treatment and research: A review of standardized assessment tools and potentially impaired populations. Clinical Psychology Review 2005; 25: 954-974.

37. Saks E, Dunn L, Marshall B, Nayak G. The California scale of appreciation: A new instrument to measure the appreciation component of capacity to consent to research. Am J Geriatr Psychiatry 2002; 10 (2); 166-174.

38. Boada M, Robles A. (eds). Análisis y reflexiones sobre la capacidad para tomar decisiones durante la evolución de una demencia: Documento Sitges. Barcelona: Glosa; 2005.

39. Ministerio de Salud. Aprueba reglamento para la internación de las personas con enfermedades mentales y sobre los establecimientos que la proporcionan. Decreto 570. Santiago: Ministerio de Salud; 2000.

40. Amor J, Amov R. Afectividad y sexualidad en las persona con deficiencia mental. Madrid: Universidad Pontificia de Comillas; 2005.

41. Eastgate G. Sexual health for people with intellectual disability. Salud pública Méx 2008; 50(2): 255-259.

42. Aunos M, Feldman M. Attitudes towards sexuality, sterilization and parenting rights of persons with intellectual disabilities. J Appl Res Intellect Disabil 2002; 15(4): 285-96.

43. Ministerio de Educación. Una mirada exploratoria de las necesidades de educación de la sexualidad, afectividad y género de niños, niñas y jóvenes con discapacidad intelectual. Santiago de Chile: Ministerio de Educación; 2006.

Recibido: 19 de noviembre de 2010

Aceptado: 12 de junio de 2012 